\title{
Expression of Ion Channels in Xenopus Oocytes
}

Alan L. Goldin

\section{1}

\section{Introduction}

Xenopus oocytes have been widely used for studying ion channels in a controlled in vivo environment since the system was initially developed for this purpose by Miledi and coworkers [1, 2]. There have been at least five major types of studies using oocytes to examine ion channel expression. The earliest use was to examine the properties of specific ion channels in a living cell free from other responses. The oocytes were injected with RNA isolated from whole brains, and the responses were analyzed using the two-microelectrode whole cell voltageclamp [2, 3], the patch clamp [4], or a variety of biochemical techniques [5, 6]. Once the responses were isolated, Xenopus oocytes were then used in a second type of study as an assay system to isolate cDNA clones encoding the proteins involved. For example, cDNA clones encoding the $5-\mathrm{HT}_{1 \mathrm{C}}$ receptor were isolated using electrophysiological assays, both by hybrid depletion [7] and by directly transcribing RNA from a cDNA library and injecting the transcripts into oocytes [8]. These types of studies are much less commonly used now because of the large number of available heterologous expression systems and cDNA clones encoding ion channels.

The third major type of study for which the Xenopus oocyte expression system has been, and continues to be, particularly useful is the correlation of molecular structure with electrophysiological function of a specific channel. The two basic approaches have been to construct defined mutations whose effects are determined by expression in oocytes, and to construct chimeric molecules between two closely related channels or receptors followed by expression in oocytes and electrophysiological analysis. These types of approach are still commonly used but with more sophisticated structural alterations and functional analyses.

The fourth general approach utilizing expression in oocytes is to determine the functional effects of mutations that cause human diseases. These types of studies are also performed using expression in other heterologous systems such as mammalian cells, and the advantages and disadvantages of each approach will be discussed in Section 1.5.3. 
The final approach that takes advantage of expression in Xenopus oocytes is to screen potential drugs to determine their relative efficacies against specific types of ion channels. These studies have been made feasible by the development of automated voltage-clamp devices. Two such devices are the Roboocyte from Multichannel Systems and the OpusXpress from Axon Instruments, which is now part of Molecular Devices. The features of these instruments are described in Section 1.4.3.

\section{2}

\section{Advantages and Disadvantages of Xenopus Oocytes}

While the Xenopus oocyte system is a valuable tool for the study of ion channel function, there are a number of important factors to consider in deciding whether oocytes are the most appropriate system to use. One of the primary advantages of oocytes is that these cells do not express a large number of ion channels and receptors, so that the exogenous protein can be studied without contamination from endogenous channels. This advantage is not true in all cases, however, as oocytes do express some channels and receptors [9]. These responses are not usually a problem for two reasons. First, only some oocytes express the channels, so that it is frequently possible to obtain oocytes that do not have endogenous current. Second, current from the injected RNA is usually much larger than that through the endogenous channels, so that it is generally possible to record the expressed current without significant contamination from native oocyte currents. In some cases, the presence of an endogenous response can be used to advantage as a second messenger system that is coupled to the initial response that is being studied.

Another advantage is that some channels can only be expressed in oocytes and not in mammalian cells. It is not possible to predict which channels fall into this category, and success using mammalian cells often depends very strongly on choosing the appropriate cell type (see Chapter 4). Even when the channels can be expressed in other systems, oocytes may still be advantageous for examination of the roles of different subunits. Since expression in oocytes involves injection of RNA, it is possible to adjust the ratio of RNA encoding each subunit and thus examine channels with a relatively well controlled composition. In contrast, it is more difficult to control the ratios of different subunits expressed by transfection in mammalian cells.

There are also some advantages to the use of oocytes with respect to electrophysiological recording. The oocyte system is particularly well suited for the study of many different mutations because injection and two-electrode voltage-clamping can be carried out rapidly and in a semi-automated fashion. In addition, studies involving modulation by second messenger systems such as phosphorylation are particularly well suited to oocytes because it is possible to express multiple proteins in the same cell, and modulators can be injected while recording with the two-electrode voltage-clamp. Finally, some analysis techniques are unique to oo- 
cytes. For example, the cut-open oocyte voltage-clamp was developed specifically for high resolution electrophysiological recording from oocytes and it is particularly well-suited for analyzing fast ionic and gating currents [10,11].

There are a number of disadvantages to using oocytes for expression of ion channels. First, as pointed out above, oocytes do express some endogenous channels and receptors. A second major disadvantage is that it has not been possible to express every channel in oocytes. On the other hand, most channels that have not been expressed in oocytes have not been expressed in other heterologous systems either, so that this problem is not unique to the use of oocytes.

Another disadvantage of oocytes is that many pharmacological agents are less potent on channels in oocytes compared to the channels in mammalian cells or native tissues. This difference in potency most likely reflects decreased accessibility of the drug because of the large number of invaginations in the oocyte membrane, the vitelline membrane surrounding the oocyte surface, or the follicle cells around the oocyte. However, although the absolute concentration of drug that is required for block is often higher than that required in native tissues, the relative efficacies of drugs against different channels are generally representative of those in native tissues. Other potential disadvantages with the use of oocytes include the need for procedures and equipment beyond that usually found in a standard research laboratory, occasional wide variations in quality due to seasonal and other factors, and the fact that they are best maintained at ambient temperature which may lead to altered synthesis and processing of mammalian channels compared to physiological conditions.

The most serious disadvantage of using expression in oocytes as an assay system is that the cells are not the native cells in which the channels are normally expressed. This can be reflected in two major ways. First, the functional properties that are observed may not be identical to those characterized in native tissues, although it is often difficult to make a direct comparison because the native cell usually contains multiple different types of channels. In addition, the functional properties may depend on the subunit composition, in which case the oocyte system can be used to determine which subunits are required for properties similar to those observed in vivo. The second consequence of oocytes not being the native tissue is that cellular trafficking is different, particularly in comparison to neurons. Because of this difference, some channels may not be expressed on the cell surface in oocytes and the effects of mutations that affect trafficking cannot be studied at all.

\section{3}

\section{Procedures for Using Oocytes}

The procedures for maintenance of Xenopus laevis, preparation of oocytes and injection with mRNA have been previously described $[12,13]$. In addition, details concerning the maintenance of Xenopus laevis and the use of oocytes can be obtained from the Xenopus Express website (http://www.xenopus.com/links.htm). 
A frog colony does not require elaborate equipment, although there are two important considerations. First, amphibians are sensitive to both chlorine and chloramine, so the water must be purified to remove both compounds. Second, although frogs can tolerate a wide range of temperature fluctuations, inconsistent temperature, and particularly elevated temperatures above $20^{\circ} \mathrm{C}$, greatly diminish oocyte viability.

Surgery to remove oocytes is a relatively simple procedure that can be carried out on a bench top in a clean room. After preparation, the follicle cells are usually removed by treatment with collagenase, although oocytes can be injected and voltage-clamped with intact follicle cells around them. Some electrophysiological responses in oocytes either depend on the presence of follicle cells or occur in the follicle cells, which would be a reason to maintain the cells. However, all procedures are technically more difficult because the follicle cells are harder to pierce and it is time-consuming to separate out individual oocytes, so it is usually best to use defolliculated oocytes.

It is possible to obtain oocytes that have been surgically removed and prepared for injection by commercial vendors. The advantages of this approach are that there is no need to maintain a frog colony, an animal use protocol is not required because no vertebrate animals will be used, and there is a considerable saving in time. The disadvantages are that the oocytes are significantly more expensive and they are only available in specific geographical areas, although the number of vendors may increase, depending on demand. One commercial source for oocytes is EcoCyte Bioscience in Germany (http://www.ecocyte.de).

For most studies, oocytes are injected with RNA that has been transcribed in vitro from a cDNA clone using either a T7 or T3 promoter. Transcription is easily performed using commercially available kits, although there are some important considerations that affect expression levels. First, the RNA needs to be capped for optimal efficiency, which is part of the procedure in most kits. Second, inclusion of Xenopus $\beta$-globin $5^{\prime}$ and $3^{\prime}$ untranslated mRNA regions and a poly(A) tail at the $3^{\prime}$ end usually enhances stability and translatability. Third, the length of the untranslated 5' region can have a dramatic effect on the level of current, with shorter regions generally resulting in greater efficiency of expression.

It is also possible to inject DNA into the nucleus of oocytes. In this case, the cDNA should be cloned downstream of a eukaryotic promoter such as the commonly used Cytomegalovirus (CMV) promoter. The advantage of injecting DNA is that there is no need to perform in vitro transcription reactions, which saves both time and money. The disadvantage is that the procedure is more difficult and requires a more sophisticated injection apparatus [12].

Cytoplasmic injection is a relatively rapid and easy procedure. The basic requirements are a dissecting microscope, a micromanipulator, and an injector that can be a simple manual or motor driven dispenser (Fig. 1.1) [12]. Alternatively, oocytes can be injected using an automated device such as the Roboocyte from Multichannel Systems, as described in Section 1.4.3.1. This instrument was developed for automated two-electrode voltage-clamping of oocytes, but it can also function for both cytoplasmic and nuclear injection of oocytes. 


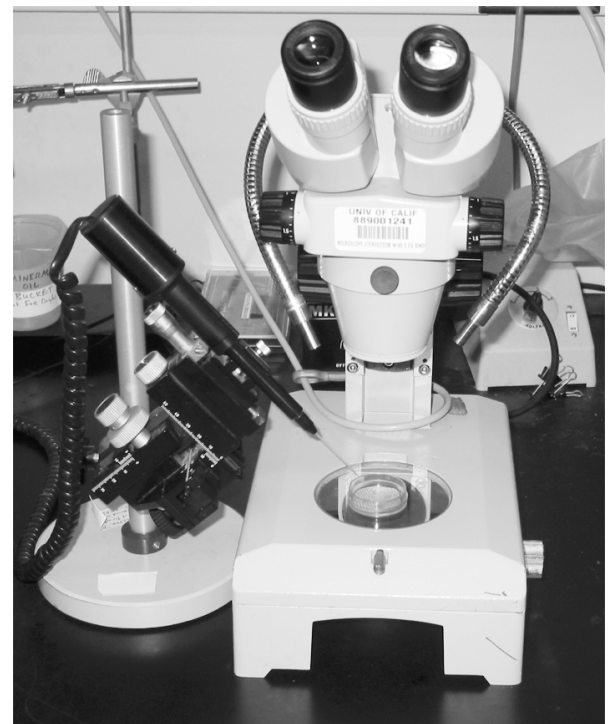

Fig. 1.1 Apparatus for cytoplasmic injection of Xenopus oocytes. The oocytes are distributed on polypropylene mesh in a 35-mm tissue culture dish on the stage of a dissecting microscope. Injection needles are made using a pipette puller to draw out the glass bores that are normally used with the microdispenser. After pulling, the needles are broken off at a tip diameter of 20-40 $\mu \mathrm{m}$, as measured with a reticle under a dissecting microscope. The injection needle is position over individual oocytes using a micromanipulator and the oocytes are injected with up to $100 \mathrm{nl}$ of RNA solution. Using these procedures, it is possible to inject 20 oocytes with one sample in a few minutes.

1.4

Types of Analyses

\subsection{1}

\section{Electrophysiological Analysis}

The most sensitive approach for analyzing ion channel function in Xenopus oocytes is the use of electrophysiology. Essentially all of the standard electrophysiological techniques can be performed on oocytes, including whole cell recording and patch-clamp recording of both macroscopic and single channel currents. In addition, techniques such as cut-open oocyte voltage-clamp recording have been developed specifically for analyzing currents in Xenopus oocytes [10]. The goal of this chapter is to present the general considerations involved in using the various approaches, as detailed procedures have been previously described [14].

\subsubsection{Two-electrode Whole Cell Voltage-clamp}

Whole cell voltage-clamping of oocytes involves using two electrodes inserted into the oocyte, rather than using one electrode to make a patch on the surface followed by rupturing the membrane, as is done in mammalian cells. One electrode is used to measure the internal potential of the oocyte and the other electrode is used to inject current (Fig. 1.2). The large size of the oocyte (about $1 \mathrm{~mm}$ in diameter and $0.5-1 \mu \mathrm{l}$ in volume for stage $\mathrm{V}$ oocytes) makes this feasible, and is both the major advantage and disadvantage of the system. One advantage is that the procedure is easy to learn and fast to perform. The electrodes are simple to pre- 


\section{Perfusion}

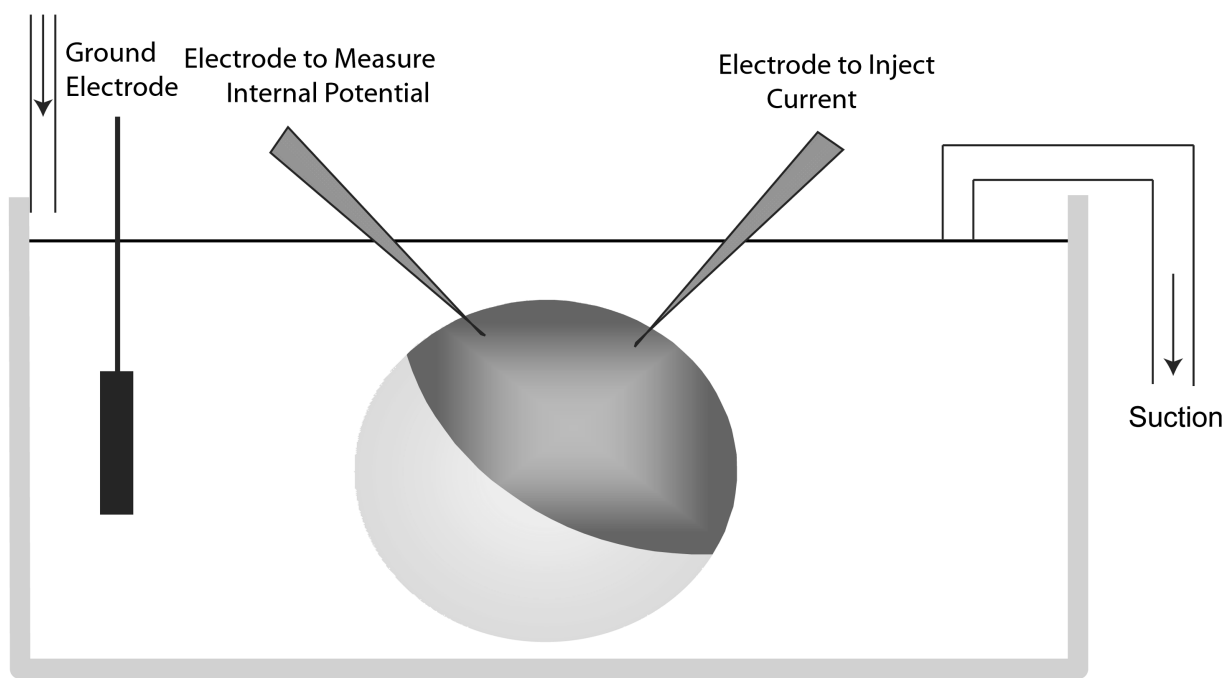

Fig. 1.2 Diagram of the two-electrode voltageclamp. The oocyte is placed in a chamber under a dissecting microscope and two electrodes are gently inserted through the membrane using micromanipulators. One electrode is used to measure the internal potential of the oocyte and the other electrode is used to inject current for clamping the oocyte at different potentials. The currents can be recorded either through the current electrode or separately through a virtual ground circuit in the bath. The bath solution can be easily and rapidly changed by continuous perfusion from gravity flow reservoirs.

pare and it is generally possible to obtain records from all oocytes if they are healthy, so that there is very little time lost in preparation. In addition, perfusion of the external medium can be easily changed multiple times. These features make the two-electrode voltage-clamp ideal for screening purposes. A second advantage is that the recordings can be stable over long periods of time, which makes it particularly useful for analyzing properties that require long protocols, such as slow inactivation. A third advantage is that it is possible to insert multiple electrodes and injection needles into the same oocyte. Therefore, modulators of channel function can be injected inside the cell while recording, so that a rapid and direct response to an intracellular signal can be observed. The final advantage of the two-electrode voltage-clamp is that it records currents through channels present in the whole cell, so that it is very sensitive. For example, currents as small as $50 \mathrm{nA}$ can be detected, which corresponds to only $5 \times 10^{4}$ molecules if the single channel current is $1 \mathrm{pA}$. The two-electrode voltage-clamp can be used to record currents over a wide range of amplitudes from about $10 \mathrm{nA}$ to over $100 \mu \mathrm{A}$, depending on the amount of RNA that is injected. However, it is important to adjust the amount of RNA to obtain currents that can be reliably clamped because it is difficult to accurately clamp the membrane potential of the oocyte if the currents are larger than $5 \mu \mathrm{A}$. 
The major disadvantage of recording from the entire oocyte is that the large size and extensive membrane invaginations result in an extremely large membrane capacitance, approximately $150-200 \mathrm{nF}$. The large capacitance causes a slow clamp settling time when the membrane potential is changed. The capacity transient can be minimized by using electrodes with low impedances of $500 \mathrm{k} \Omega$ or less. This can be accomplished by filling the electrode tips with low-melting temperature agarose, making it possible to have a large tip opening without significant leakage of $\mathrm{KCl}$ into the oocyte [15]. However, even with the best electrodes it is difficult to obtain data during the first $1-2 \mathrm{~ms}$ of a depolarization, which is the time during which rapidly activating voltage-gated channels such as sodium channels are activated (Fig. 1.3). The large capacity transient is not a problem when recording slow responses or ligand-gated currents that do not require changes in voltage. A second major disadvantage is that there is no control of the internal cellular environment, so that it is difficult to perform quantitative studies, for example examining selective permeability.

\subsubsection{Cut-open Oocyte Voltage-clamp}

The cut-open oocyte voltage-clamp was developed to circumvent many of the disadvantages involved in using the two-electrode whole cell voltage-clamp [10, 11]. In this procedure, the oocyte is inserted in a chamber that separates the surface into three regions (Fig. 1.4). The top portion of the oocyte membrane is the region that is clamped and is the section from which currents are actually recorded. The middle portion is a guard that is clamped to the same potential as the top to null leakage currents though the seals. The bottom portion is the region of the oocyte that is "cut-open", either by permeabilization with saponin or by insertion of a cannula, thus making it possible to perfuse the internal environment and to inject current intracellularly through a low resistance pathway. The internal environment is clamped to ground, as measured by a low resistance electrode inserted through the top of the oocyte, which ensures that the region of the oocyte near the top portion is accurately held at ground. The bath surrounding the top portion of the oocyte is clamped to the command potential, which can be rapidly changed with minimal series resistance. Currents are recorded through low resistance electrodes in the top chamber.

There are a number of advantages to the cut-open oocyte voltage-clamp compared to the two-electrode voltage-clamp. First, the capacity transient is minimized so that the clamp can settle in $50 \mu \mathrm{s}$, which is fast enough to study activation of even the fastest ion channels (Fig. 1.3). Second, current noise is quite low, approximately $1 \mathrm{nA}$ RMS at $5 \mathrm{kHz}$ bandwidth. Third, it is possible to control the solutions in both external and internal environments. The internal solution can be accurately adjusted with the initial perfusate, but is difficult to change because of the slow perfusion rate, even when using a cannula. In contrast, the external solution can be changed quickly and completely. Finally, the recordings can be stable for hours. These advantages make the clamp particularly well suited for studies involving fast ionic and gating currents. 
TWO-ELECTRODE VOLTAGE CLAMP

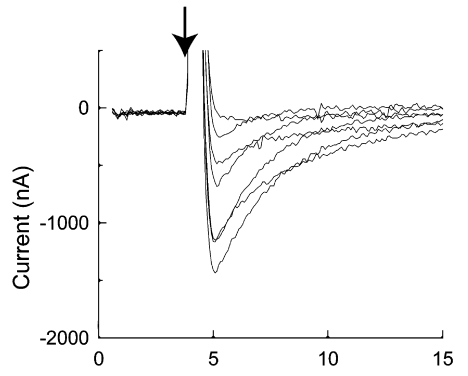

\section{CUT-OPEN OOCYTE VOLTAGE CLAMP}

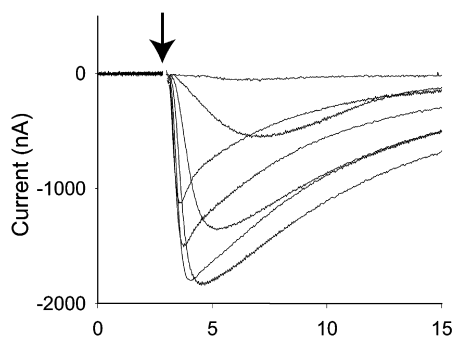

MACROPATCH VOLTAGE CLAMP

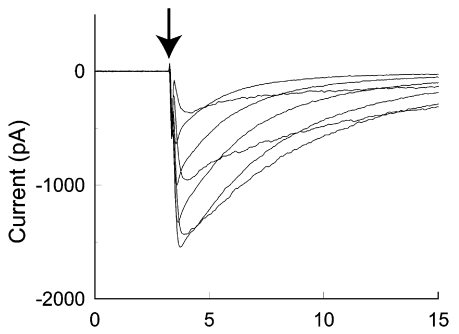

PATCH CLAMP (SINGLE CHANNELS)

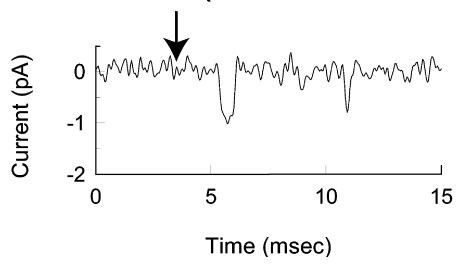

Fig. 1.3 Representative traces of sodium currents using the different recording techniques. The two-electrode voltage-clamp records from channels throughout the entire oocyte membrane, which results in a large capacitive transient (the gap in the current records) so that data cannot be obtained for 1-2 ms after a depolarization. The cut-open oocyte voltage-clamp records from channels in approximately a third of the oocyte membrane. Therefore, the magnitudes of the currents are comparable, but the time resolution is significantly faster because of the design of the clamp. The macropatch voltage-clamp records from channels in a small patch of oocyte membrane, resulting in fast time resolution but smaller current amplitudes (note the current scale is in $\mathrm{PA}$ rather than $\mathrm{nA}$ ). Small patches can be used to record single channel activity with excellent time resolution. The amplitude of single sodium channels is approximately $1 \mathrm{pA}$ at the potential used for these recordings. All of the data were obtained from oocytes injected with RNA encoding the rat $\mathrm{Na}_{\mathrm{v}} 7.2$ sodium channel $\alpha$ subunit alone. The macroscopic current traces are shown for depolarizations from a holding potential of $-100 \mathrm{mV}$ to a range of potentials between -30 and $+30 \mathrm{mV}$ in $10 \mathrm{mV}$ increments. The single channel current trace is shown for a depolarization from -100 to $-30 \mathrm{mV}$. The arrows indicate the start of the depolarization.

The major disadvantages of the cut-open oocyte voltage-clamp procedure are that it requires specialized equipment and that it is more difficult to use than the twoelectrode voltage-clamp. However, all of the equipment for this procedure, including the voltage-clamp, recording chamber and temperature controller, are commercially available from Dagan Corporation (http://www.dagan.com/ca-1b.htm). 


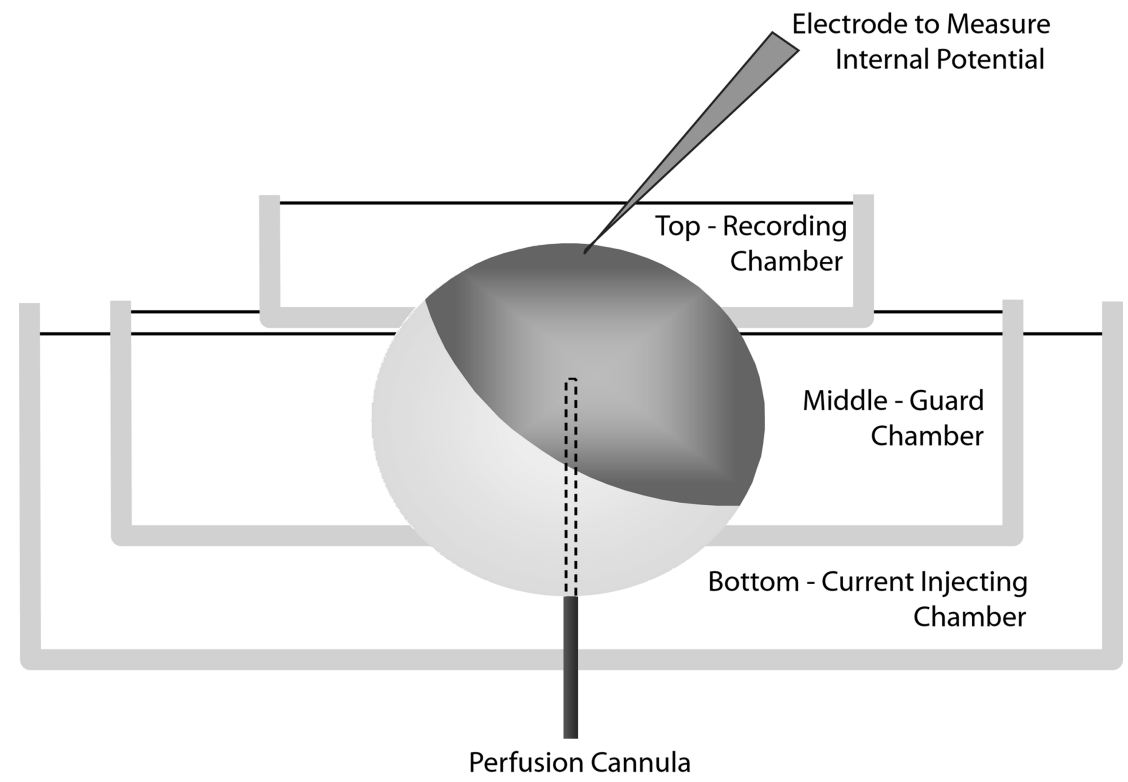

Fig. 1.4 Diagram of the cut-open oocyte voltage-clamp. The oocyte is inserted in a chamber that separates the surface into three regions. The top chamber is clamped to the command potential and is the section from which currents are recorded. The middle chamber is a guard that is clamped to the same potential as the top to null leakage currents though the seals. The bottom chamber is used to inject current intracellularly through a low resistance pathway. The internal environment is clamped to ground as measured by a low resistance electrode inserted through the top of the oocyte. A cannula inserted into the oocyte through the bottom chamber makes it possible to perfuse the internal environment.

\subsubsection{Macropatch Clamp}

An alternative method of circumventing the problems caused by the large size of the oocyte is to record from only a fraction of the membrane in an isolated patch [16]. To record macroscopic currents, an electrode with a relatively large opening of about $10 \mu \mathrm{m}$ in diameter is used to establish a macropatch on the surface membrane (Fig. 1.5). Recording can be performed in either the cell-attached mode or excised inside-out mode. The cell-attached mode is technically easier and maintains the normal cytoplasmic environment, but the intracellular potential needs to be determined. This potential can be measured by inserting electrodes into the oocyte, which is possible because of its large size. Either a single electrode can be used to measure the potential, or two electrodes can be used to clamp the oocyte at a fixed holding potential. The use of two electrodes has the advantage that channels throughout the remainder of the oocyte are held at the desired potential, minimizing slow inactivation of voltage-gated channels so that the same oocyte can be used for multiple patches. The excised patch technique allows complete control of the potential on both sides of the membrane, but it can be more diffi- 
MACROPATCH RECORDING
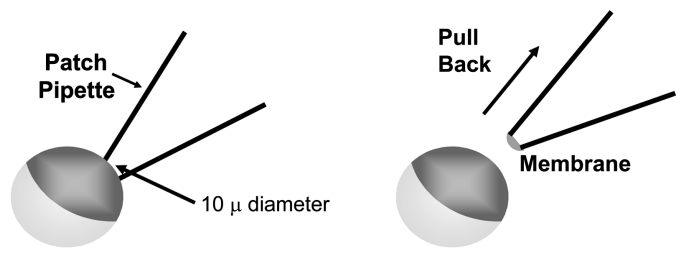

SMALL PATCH RECORDING

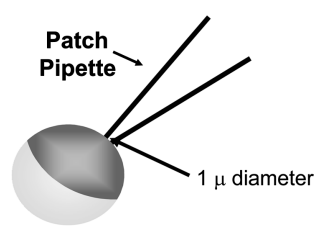

Cell-Attached Patch

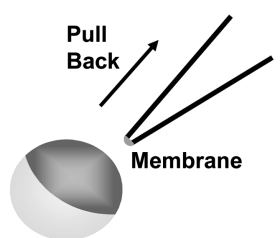

Excised Patch
Fig. 1.5 Macropatch versus small patch recording. To record macroscopic currents using the patch clamp, an electrode with a large tip diameter of approximately $10 \mu \mathrm{m}$ is used to make a seal with the oocyte membrane. Currents can be recorded while the electrode is still attached to the intact oocyte or the electrode can be gently pulled back to record currents through an excised portion of the membrane. More sensitive recordings can be obtained using the same approaches but with a smaller diameter electrode tip (approximately $1 \mu \mathrm{m})$.

cult to perform because the seal between the membrane and the large diameter electrode is less stable than with a small electrode. An advantage of using an excised patch is that the internal face of the membrane can be perfused with different solutions.

There are a number of advantages in using the macropatch technique to record from ion channels in oocytes. First, the capacity transient is minimized because only a small region of the membrane is depolarized, which makes it possible to record fast ionic and gating currents (Fig. 1.3). Second, there is complete control of the solutions on both sides of the membrane if the patch is excised, although only the internal side can be altered with perfusion because the outside is fixed by the electrode composition. These characteristics of the macropatch technique are similar to those of the cut-open oocyte voltage-clamp. An advantage compared to the cut-open oocyte technique is that the equipment is not as specialized, so that a patch clamp amplifier from any supplier can be used.

There are some disadvantages in using the macropatch technique compared to the cut-open oocyte voltage-clamp. First, macropatches are usually not as stable as oocytes in the cut-open oocyte voltage-clamp. The decreased stability is a function of the patch and the fact that it is necessary to remove the vitelline membrane from the oocyte to make a seal with the electrode. Oocytes without the vitelline membrane are less stable than intact oocytes and they will lyse if exposed to air. A second disadvantage of macropatch recording is that it usually requires a high level of expression in the oocyte. In this regard, it is possible to use smaller electrodes by increasing the level of expression, which in turn decreases the technical difficulty. Because the macropatch technique involves either cell-attached or excised inside-out patches, there is no access to the external surface of the portion of the membrane being studied. Therefore, macropatch recording is 
not well suited for studying the interactions of toxins that directly bind to a channel from the external surface. On the other hand, it is an excellent system for studying modulation through second messenger systems, particularly in the cellattached mode.

\subsubsection{Single Channel Analysis}

Xenopus oocytes can also be used for conventional patch clamp recording, including single channel analysis. This approach differs from macropatch recording only in the size of the electrode, with a tip diameter of about $1 \mu \mathrm{m}$ compared to about $10 \mu \mathrm{m}$ for macropatches (Fig. 1.5). To make the seal, the vitelline membrane must first be removed from the oocyte, which is accomplished by placing the oocyte in a hypertonic solution $(200 \mathrm{mM} \mathrm{NaCl})$. The oocyte shrinks, leaving the vitelline membrane exposed so that it can be manually removed with forceps. Patches can then be obtained in the cell-attached or excised configuration, as in mammalian cells. One disadvantage of the cell-attached mode is that the intracellular potential must be determined. However, an advantage of using oocytes for this purpose is that additional electrodes can be inserted into the large oocyte, making it possible to accurately measure or clamp the intracellular potential. On the other hand, the additional electrodes and voltage-clamp increase the noise, which can be a significant problem for single channel recording. A second disadvantage of cell-attached recording is that oocytes express a high level of endogenous stretchactivated channels [17], and currents through these channels may interfere with the signal of interest.

The patches can be excised in either the inside-out or outside-out configuration, just as with mammalian cells (Fig. 1.6). A difference compared to mammalian cells is that it is more difficult to rupture the oocyte membrane compared to a mammalian cell membrane. Positive pressure is generally the most effective technique, particularly with electrodes that have small electrode tip openings. In addition, the oocyte cannot be clamped with a single electrode after the membrane has been ruptured, unlike the situation with mammalian cells. Once the patch is excised, recording is comparable to recording from mammalian cell patches.

Single-channel analysis in oocytes is generally equivalent to single channel analysis using mammalian cells, and it therefore has the same advantages and disadvantages. First, there is excellent time resolution because only a small portion of the membrane is depolarized. Therefore, it is possible to obtain detailed information about the opening and closing of individual channel molecules, which provides the most quantitative information for developing gating models (Fig. 1.3). The major disadvantage is that it is technically difficult to get high quality data, especially with small conductance channels. In addition, single channel recording is very time consuming for both acquisition and analysis.

There are also some advantages and disadvantages to using oocytes for this purpose compared to mammalian cells. One advantage is that the level of expression can be adjusted by injecting different quantities of RNA. Therefore, it is possible to maximize the probability of obtaining patches that contain single channels. A 


\section{INSIDE-OUT PATCH}
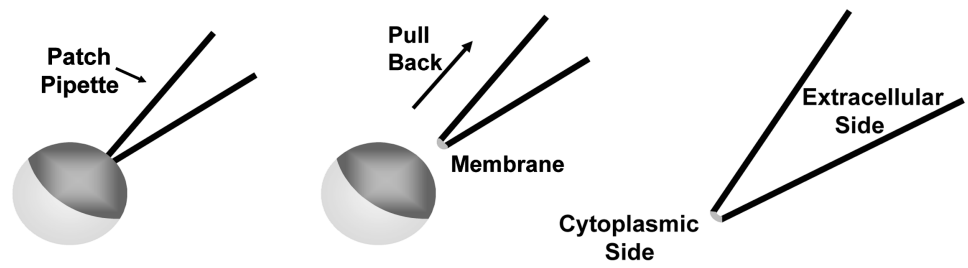

\section{OUTSIDE-OUT PATCH}

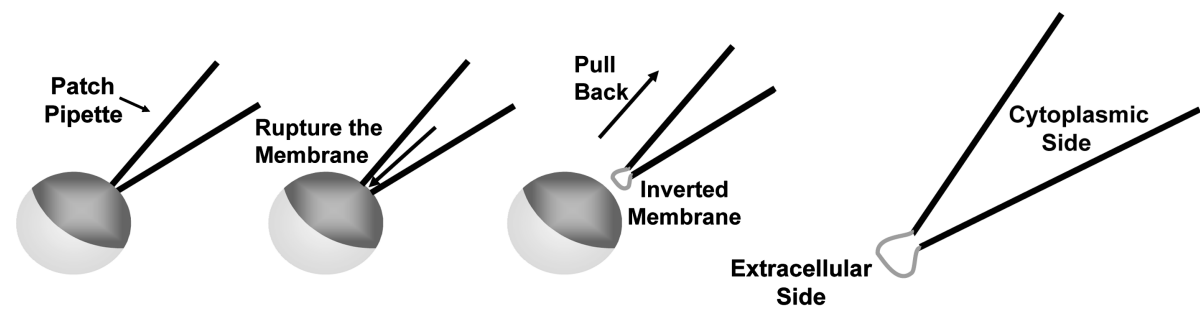

Fig. 1.6 Inside-out versus outside-out patch recording. Patches of oocyte membrane can be excised in either the inside-out or outside-out configuration. In both cases, the electrode is first placed against the oocyte membrane to obtain a tight seal. For an inside-out patch, the electrode is then gently pulled back,

leaving the cytoplasmic face of the membrane exposed to the bath solution. For an outsideout patch, the oocyte membrane is first ruptured, after which the electrode is gently pulled back. The membrane reforms a vesicle attached to the electrode with the external face exposed to the bath solution.

related advantage is that the oocytes can be screened first using a two-electrode voltage-clamp to determine the levels of expression so that only cells with an appropriate level of current are used to obtain patches. On the other hand, a disadvantage of oocytes is that the vitelline membrane must be removed before patching, which adds an extra step and decreases the viability of the oocyte.

\subsection{2}

\section{Biochemical Analysis}

Ion channels expressed in oocytes can be studied using standard biochemical techniques such as immunoprecipitation or binding assays [18, 19]. The major disadvantage of these techniques in oocytes is that they are significantly less sensitive than the electrophysiological approaches. Using the whole cell voltage-clamp, it is possible to detect as few as $10^{5}$ channel molecules in a single oocyte (less than $10^{-18}$ mole). On the other hand, biochemical techniques are generally reliable down to the level of $10^{-12}$ mole, although this depends strongly on the specific activity of the reagents being used. Because of this limitation, it is necessary to express the channels at a much higher level for biochemical analysis than is required for electrophysiological recording. 
Another consideration in using biochemical techniques to analyze ion channel expression in oocytes is that solubilization results in isolation of both cytoplasmic and membrane proteins. Functional ion channels are located in the membrane, but there is generally a large intracellular pool of molecules [20]. An advantage of electrophysiological analysis is that it examines only the functional channels in the membrane, whereas immunoprecipitation of total oocyte proteins examines both membrane-bound and cytoplasmic proteins. This problem can be mitigated by using membrane preparations for solubilization [21], but it is difficult to remove all nonmembrane proteins and those bound to internal membranes. Ligand binding assays do not suffer from this limitation and thus they are well suited for the study of ion channels expressed in oocytes.

Ligand binding assays using oocytes are very similar to those carried out using mammalian cells (see Chapter 7). The advantage of using oocytes is that it is possible to easily and quickly express many different channel mutations or variations. In addition, the assay can usually be carried out on a single oocyte so that the composition of channels and subunits is relatively homogenous. The use of a single cell is also the major disadvantage of oocytes, in that it is necessary to obtain a sufficiently high level of expression so that the ligand can be detected. Therefore, the assay requires a high affinity ligand that can be labeled to high specific activity.

\section{4 .3}

\section{Compound Screening}

An application of Xenopus oocyte expression that has become more common in the past few years is screening the effectiveness of new pharmaceuticals and those in development. In this regard, the use of oocyte expression cannot really be considered high throughput screening but rather, moderate throughput screening. Even though the limitation on throughput is unlikely to be eliminated anytime soon, there are some advantages to using oocyte expression for this purpose.

A major advantage of screening in oocytes is that the assay is electrophysiological, which is the most detailed and relevant response to the compound or drug. In this regard, screening ion channels in oocytes is comparable to screening with one of the automated patch clamp systems that have been developed. Because automated patch clamps are designed for screening cell lines that are usually constructed to be stably expressing the genes of interest, oocytes are better suited for analyzing multiple channel variations such as mutations or different compositions of subunits. For this reason, the oocyte system is particularly appropriate for target identification and optimization. There are also some situations for which the oocyte system has a clear advantage. The first is if the channels do not express well (or at all) in mammalian cells. The second is if the mammalian cells express native currents that interfere with detection of the expressed response.

A major disadvantage of using oocytes for screening drugs is that the cell is not the physiological target and the responses may differ from those that occur in vivo. However, this is a criticism of most screening systems because the physiological target cells often cannot be used because they express many different chan- 
nels and receptors. One difference between oocytes and mammalian cells is that higher drug concentrations are often required for effective block of ion channels in oocytes compared to mammalian cells. This difference probably reflects limited drug access to the oocyte membrane because of the large number of invaginations. On the other hand, although the actual $\mathrm{EC}_{50}$ is usually higher in oocytes, the rank order of different drugs on the same channel or the relative efficacy of the same drug on different channels is likely to reflect the situation with native tissue.

Two different automated systems have been developed for electrophysiological screening of ion channels using Xenopus oocytes. The Roboocyte system uses a serial approach in which oocytes are tested sequentially, and the OpusXpress system uses a parallel approach in which eight oocytes are tested simultaneously.

\subsubsection{Serial Recording Using the Roboocyte}

The Roboocyte was developed by Multichannel Systems for the automated screening of oocytes in a serial fashion. Information about the Roboocyte can be obtained from the company web site at the following address: http://www.multichannelsystems.com/products/roboocyte/robointro.htm.

The instrument consists of a single head that moves vertically for both injection and recording, with the oocytes located in the chambers of 96 well dishes (Fig. 1.7). The head can be configured with an injection needle or with a recording assembly that contains both voltage and current electrodes and a perfusion needle. The dish sits in a carrier that moves in the $X$ and $Y$ directions to position each oocyte sequentially under the head. The entire instrument is computer-controlled with separate procedures for injection and electrophysiological recording. Perfusion can be controlled using a gravity based system that is part of the apparatus and contains either 8 or 16 valves, or the device can be connected to a Gilson liquid handler that can dispense compounds from multiwell plates.

The Roboocyte is ideally suited to a situation in which the same sample will be injected into every oocyte, with alterations in the recording conditions or drug application. A major advantage is that injection can be automated after a single setup configuration. On the other hand, it is not possible to inject different samples without going through the complete set-up procedure again. Oocytes can be obtained already prepared in multiwell plates from EcoCyte Bioscience (http:// www.ecocyte.de), although the availability is limited to specific geographic regions.

A unique advantage of the Roboocyte is that it is relatively simple to perform nuclear injection. For this purpose, oocytes are allowed to settle in the 96 well plates, during which time the lighter nucleus rises towards the top surface of the oocyte. Because the oocytes are injected vertically, the instrument can be configured so that the needle pierces the nucleus by increasing the depth of injection. Nuclear injection avoids the time and expense of in vitro transcription. The coding region is cloned following a eukaryotic promoter rather than a T7 or T3 promoter, and the RNA is transcribed in the oocyte nucleus. A disadvantage of nuclear injec- 


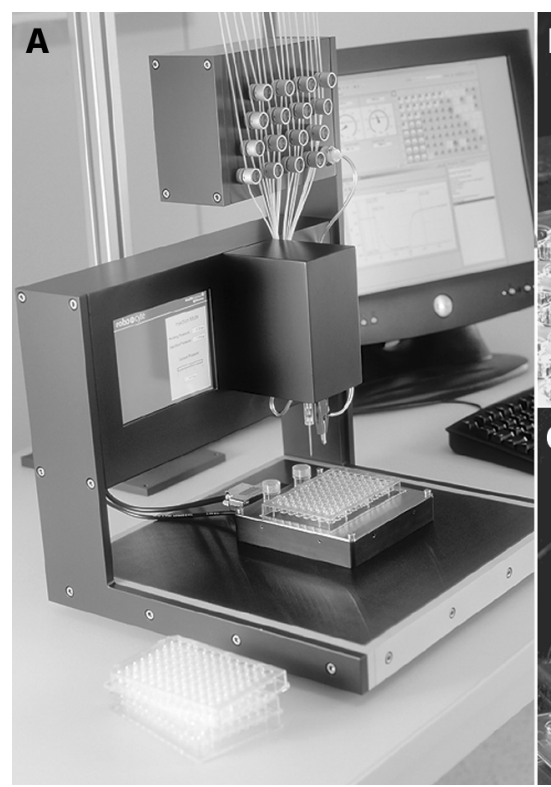

Fig. 1.7 The Roboocyte automated voltageclamp from Multichannel Systems. A, The instrument consists of a single head that moves vertically for both injection and recording, with the oocytes located in the chambers of a 96 well dish that moves on a cushion of pressur-

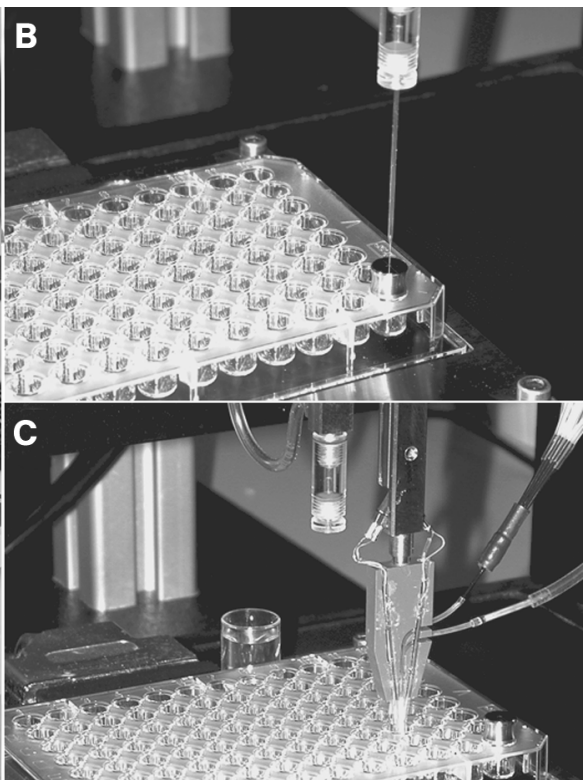

ized air above a magnetic steel plate. Perfusion can be controlled with a gravity-based system containing 16 valves. B, Close-up view of an injection needle. C, Close-up view of the recording head, which contains both voltage and current electrodes and a perfusion needle.

tion is that it is difficult to control the amount of RNA that is synthesized and hence the size of the current that is expressed. In addition, it is impossible to inject fixed ratios of different subunits.

The Roboocyte performs automated two-electrode electrophysiological recording with semi-automated on-line and off-line analysis. The recording protocol is the same for each oocyte, and the oocytes are tested for viability before recording so that data are not obtained from dead oocytes. The sampling frequency is up to $2-\mathrm{kHz}$, which is a lower time resolution than that of the OpusXpress. The perfusion system that is included as part of the Roboocyte is limited to a maximum of 16 samples flowing by gravity. The manifold includes the outlet from all reservoirs so there is no lag time for perfusion, but there is a risk of cross-contamination at the tip. The instrument is designed to interface with a more sophisticated liquid handling system from Gilson, in which case samples can be stored in a variety of wells or tubes and the flow rate is controlled by a peristaltic pump. A disadvantage of this system is that there is a significant lag time for drug delivery to the recording chamber, so that the flow rate must be calibrated to determine when the compound reaches the oocyte. 


\subsubsection{Parallel Recording Using the OpusXpress}

The OpusXpress was developed by Axon Instruments, which is now part of Molecular Devices. This instrument is designed for automated analysis of oocytes in a parallel configuration. Information about the OpusXpress can be obtained from the company web site at the following address: http://www.axon.com/cs_Opus Xpress.html

The instrument consists of eight individual recording chambers that are configured with perfusion and ground assemblies (Fig. 1.8). Separate voltage and current electrodes are positioned in each chamber for a total of 16 electrodes. Manipulation of the electrodes is controlled by 8 separate controllers and recording is carried out using 8 separate voltage-clamp modules. As with the Roboocyte, the entire instrument is computer-controlled. Perfusion is applied from one of two large reservoirs to all of the chambers simultaneously. Individual compounds are applied from 96 well dishes by an automated liquid handling system that uses 8 parallel pipette tips. The OpusXpress is designed purely as a recording instrument with no provision for automated injection, so that injection of the oocytes must be carried out separately.

The OpusXpress is particularly well suited for examining oocytes injected with different types of RNA. The parallel design has the potential to increase throughput, as recordings are obtained from 8 oocytes simultaneously. The initial set-up time is longer than for the Roboocyte because 16 electrodes must be prepared and positioned in the holders. However, the electrodes can be reused for a number of days, so that the set-up for additional recordings only involves replacing the
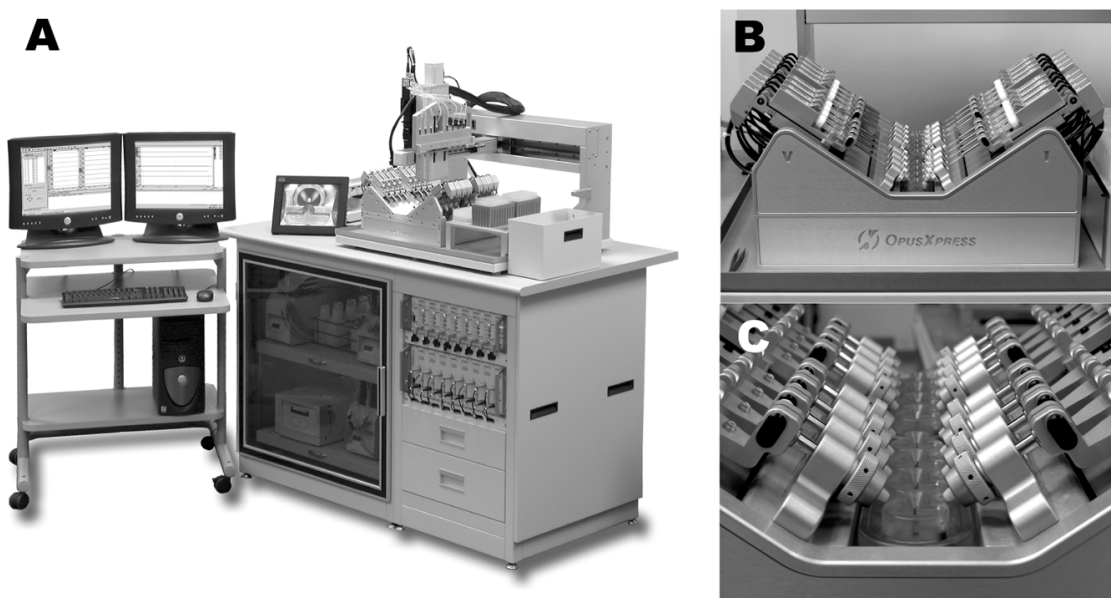

Fig. 1.8 The OpusXpress automated voltageclamp from Molecular Devices. A, The instrument consists of eight individual two-electrode voltage-clamps and a liquid handling system. $B$, Close-up view of the automated manipulators that control the 8 pairs of electrodes, with the voltage electrodes on the left and the current electrodes on the right. C, Close-up view of the 8 voltage-clamp recording chambers, each of which is equipped with perfusion and virtual ground assemblies. 
oocytes, which takes significantly less time. A major advantage of this approach is that compounds can be applied simultaneously to oocytes expressing different channels.

Recordings are obtained using the same series of protocols for all 8 oocytes, with automated operation and real-time analysis. In addition, oocytes are tested for viability so that compounds are not delivered to chambers containing dead oocytes. An advantage of the OpusXpress is that the sampling frequency is up to $30 \mathrm{kHz}$, which is significantly faster than for the Roboocyte. The instrument can be programmed for a variety of recording protocols and solutions exchanges. The compounds are loaded into the chambers of 96 well dishes and relatively small volumes are required. Another advantage of the OpusXpress is that each compound is delivered via an individual pipette tip, so that there is no delay and no cross-contamination.

\section{5}

\section{Examples of Use}

\subsection{1}

\section{Characterization of cDNA Clones for a Channel}

Originally, the oocyte expression system was especially useful for the isolation of cDNA clones encoding ion channels for which no sequence information was available. With the acquisition of complete genomic sequence information from many species, new ion channels are now usually identified as candidate genes based on their similarity with known family members. However, once the sequence has been determined, it is still necessary to demonstrate that the gene encodes a functional channel. This step is critical because the sequence may not correctly predict the properties of the encoded protein. An example of this situation is the characterization of the BSC1 channel from the German cockroach Blattella germanica [22]. BSC1 was originally identified as the orthologue of the DSC1 channel from Drosophila melanogaster [23], which was in turn identified by its sequence similarity to voltage-gated sodium channels [24]. However, neither DSC1 nor BSC1 had been functionally expressed, so that the assignment of these genes as voltagegated sodium channels was based purely on sequence similarity.

Zhou et al. [22] succeeded in expressing BSC1 in oocytes and demonstrated that it encodes a functional voltage-gated cation channel whose properties differ significantly in a number of ways from those of voltage-gated sodium channels. First, the channels are more selective for barium than for sodium. Second, the kinetics of activation and inactivation are significantly slower than the kinetics of sodium channel gating. Third, the channels deactivate very slowly with a substantial tail current. Finally, sodium currents through the channel can be blocked by low concentrations of calcium, resulting in an anomalous mole fraction effect. All of these properties are more similar to voltage-gated calcium channels than to voltagegated sodium channels. BSC1 appears to be the prototype of a novel family of in- 
vertebrate voltage-dependent, cation channels with a close structural and evolutionary relationship to voltage-gated sodium and calcium channels.

The Xenopus oocyte expression system was both helpful and problematical for the characterization of BSC1. A critical advantage was that the BSC1 channel had only been expressed in oocytes, so that this system was essential to study the channel. Second, currents through BSC1 were too small to measure using isolated patch recording, which made it difficult to compare the permeability of different ions. The cut-open oocyte voltage-clamp made it possible to examine permeability because the ionic composition on both sides of the membrane could be altered while still recording macroscopic currents through most of the cell membrane. The major disadvantage of using oocytes was that they express a robust calciumactivated chloride current that was turned on by calcium entry through BSC1. The chloride current made it significantly more difficult to record calcium current through the slowly gating BSC1 channel, which made it necessary to record barium rather than calcium current.

\section{5 .2}

\section{Structure-Function Correlations}

One of the most powerful uses for oocytes in the study of ion channels has been to correlate molecular structure with biochemical and electrophysiological functions. Studies of this type initially involved mutagenesis followed by relatively straightforward analysis using either the two-electrode voltage-clamp or patch clamp. These approaches identified many of the regions involved in activation [25, 26], inactivation [27-30], toxin-binding [31-34] and permeation [35, 36] of voltagegated sodium and potassium channels. More sophisticated approaches have since been developed that take advantage of the features of oocyte expression to investigate the molecular mechanisms involved.

One technique that has been particularly powerful has been the combination of fluorescent microscopy with electrophysiological recording to determine the movement of specific regions of the channel. This approach utilizes the substituted cysteine scanning accessibility method originally developed by Javitch et al. [37], which involves replacing amino acids individually with cysteine and then using cysteine-modifying reagents to determine the accessibility of those residues (see Chapter 2). For the fluorescence measurements, the cysteine residue is labeled with a fluorophore that can be used to detect movement of the specific region of the molecule. While cysteine scanning mutagenesis has been performed using a variety of expression systems, the combination of fluorescence microscopy with electrophysiological recording is a unique capability of the oocyte expression system. These approaches have been developed and used extensively by Isacoff and coworkers and Bezanilla and coworkers.

The combination of fluorescence and electrophysiology measurements has revealed a great deal about the movement of the voltage sensor in the potassium channel. Cha et al. [38] used lanthanide-based resonance energy transfer to measure the voltage-dependent distance changes near the S4 subunit of the Shaker 
potassium channel, demonstrating that gating is accompanied by a rotation and possible tilt rather than a large transmembrane movement. Mannuzzu et al. [39] used voltage-clamp fluorometry of oocytes to measure gating rearrangements in the Shaker potassium channel. Their results demonstrated that there are two charge-carrying steps, the first of which takes place independently in each subunit whereas the second involves cooperative interactions between S4 segments. This approach has also been useful for testing structural models of voltage-gated ion channels. For example, Gandhi et al. [40] used accessibility probing and disulfide scanning experiments to demonstrate that the S4 voltage sensor in the bacterial $\mathrm{K}_{\mathrm{v}} \mathrm{AP}$ potassium channel lies in close apposition to the pore domain in the resting and activated state, in contrast to the predictions of the crystal structure for that channel [41, 42].

Similar approaches have revealed details about the interactions of the four S4 voltage sensors in the sodium channel. Chanda et al. [43] used the cut-open oocyte voltage-clamp to simultaneously record fluorescence signals and gating currents, demonstrating that the voltage-dependent movement of the S4 segment in domain IV is a late step in the activation process after the S4 segments in domains I-III have moved. They further showed that the S4 segment of domain III most likely moves at the most hyperpolarized potentials and that the S4 segments in domains I and II move at more depolarized potentials. Chanda et al. [44] used the same approach to provide direct evidence for coupling interactions between the voltage sensors. Their results indicate that movement of all four voltage sensors is coupled to varying extents, with energetic linkage between the voltage sensors in domains I and IV.

The technology has been continually improved in various ways. Sonnleitner et al. [45] used total internal reflection fluorescence microscopy, which allowed them to measure the movement of single voltage-gated Shaker potassium channels rather than the movements of large ensembles of proteins. Asamoah et al. [46] utilized a novel fluorescent probe (Di-1-ANEPIA) to record dynamic changes in the electric field during the gating process of the Shaker potassium channel. Cohen et al. [47] developed a novel fluorescent probe (aminophenoxazone maleimide), which made it possible to track the motions of the side chains to which the probe was attached. These approaches have provided very detailed mechanistic and structural information about the movements of specific regions of ion channels, and they are uniquely suited to expression in Xenopus oocytes.

\section{5 .3}

\section{Studies of Human Disease Mutations}

The oocyte expression system has been extensively used to characterize the effects of ion channel mutations that cause human diseases. An example of this use is the study of mutant voltage-gated sodium channels that cause diseases of the musculoskeletal, cardiovascular and nervous system. Mutations in the SCN4A gene encoding the $\mathrm{Na}_{\mathrm{v}} 1.4$ skeletal muscle sodium channel cause three neuromuscular diseases, periodic paralysis, paramyotonia congenita and the potassium-ag- 
gravated myotonias $[48,49]$. Mutations in the SCN5A gene encoding the $\mathrm{Na}_{\mathrm{v}} 1.5$ cardiac channel cause long QT type 3, which predisposes to ventricular tachycardia (torsades de pointes), and Brugada syndrome, which is manifested as ventricular fibrillation [50]. Mutations in any of three neuronal sodium channel genes cause generalized epilepsy with febrile seizures plus (GEFS+). The mutations have been identified in $S C N 1 A$ encoding $\mathrm{Na}_{\mathrm{v}} 1.1$ [51-53], SCN2A encoding $\mathrm{Na}_{\mathrm{v}} 1.2$ [54] and $S C N 1 B$ encoding the $\beta 1$ subunit $[55,56]$.

These mutations have been analyzed using a variety of different expression systems, each of which has certain advantages and disadvantages. Most studies of the mutations in the skeletal muscle sodium channel have been carried out using transfected HEK or tsA201 cells [57, 58], although some studies have been carried out using oocytes [59]. However, neither expression system is a very good model for skeletal muscle fibers, in which the mutant channels are expressed in vivo. In fact, Cannon et al. [60] used theoretical reconstructions to demonstrate that the integrity of the muscle cell T-tubule system is required to produce myotonia. Similarly, mutations in the cardiac sodium channel have been studied using both oocytes [61-63] and mammalian cells [63, 64], with the same reservation that neither system is a good model for cardiac myocytes. Papadatos et al. [65] solved this problem by constructing mice in which the mouse $\operatorname{Scn} 5 a$ gene encoding the $\mathrm{Na}_{\mathrm{v}} 1.5$ cardiac sodium channel was disrupted, which made it possible to study both the electrophysiological properties of the ventricular myocytes and the electrocardiographic characteristics of the mice.

Similar studies have been carried out to analyze the effects of mutations causing GEFS+ using both oocytes [66-69] and mammalian cells [70, 71]. The results in the two different systems are sometimes comparable and sometimes different. For example, using the oocyte expression system, Spampanato et al. [66, 67] demonstrated that $\mathrm{R} 1648 \mathrm{H}$ dramatically accelerates recovery from inactivation, W1204R shifts the voltage-dependence of activation and inactivation in the negative direction, and T875M enhances slow inactivation. These results suggest that multiple different alterations in sodium channel function can lead to a similar seizure phenotype. Lossin et al. [70] examined the same three mutations using an HEK cell expression system and obtained different results. They observed a marked increase in persistent current for $\mathrm{R} 1648 \mathrm{H}$ and a slight increase in persistent current for T875M and W1204R, and they hypothesized that the epileptic phenotype resulted from the persistent current in all cases. It is not known which alterations reflect the actual effects of the mutations in neuronal cells in vivo. These discrepancies emphasize the necessity to examine the effects of diseasecausing mutations in the cell types in which they are normally expressed in vivo.

There are certain instances in which the oocyte expression system is particularly well suited for the analysis of a disease-causing mutation. One mutation that causes GEFS+ is D1866Y, which alters an evolutionarily conserved aspartate residue in the C-terminal cytoplasmic domain of the sodium channel $\alpha$ subunit [72]. This mutation decreases modulation of the $\alpha$ subunit by $\beta 1$, which normally causes a negative shift in the voltage-dependence of inactivation in oocytes. There is less of a shift with the mutant channel, resulting in a $10 \mathrm{mV}$ difference between 
the wild-type and mutant channels in the presence of $\beta 1$. This shift increases the magnitude of the window current, which results in more persistent current during a voltage ramp. Computational analysis suggests that neurons expressing the mutant channels would fire an action potential with a shorter onset delay in response to a threshold current injection, and that they would fire multiple action potentials with a shorter inter-spike interval at a higher input stimulus. The results suggest that the D1866Y mutation weakens the interaction between the $\alpha$ and $\beta 1$ subunits, demonstrating a novel molecular mechanism leading to seizure susceptibility. The use of oocytes made it possible to quantitatively assess the effects of $\beta 1$ by injecting different ratios of RNA encoding the $\alpha$ and $\beta 1$ subunits, which is very difficult to accomplish using a mammalian cell expression system.

\section{6}

\section{Conclusions}

In summary, Xenopus oocytes have been widely used as a heterologous expression system for the study of ion channels. Most channels can be expressed in a variety of different cell types, each of which has its own advantages and disadvantages. Oocytes are particularly well suited for studying many different samples, such as multiple mutations or the effects of different compositions of subunits. In addition, they are excellent for correlating structure with function using a combination of molecular biological and electrophysiological techniques, some of which have been developed specifically for oocytes. Finally, oocytes represent the only heterologous system in which some channels have been expressed. On the other hand, oocytes are not the native cells in which the channels are normally expressed, and this caveat must be remembered when interpreting the results.

\section{Acknowledgments}

Work in the author's laboratory is supported by grants from the NIH (NS48336), the National Multiple Sclerosis Society (RG3405) and The McKnight Endowment Fund for Neuroscience (34653).

\section{References}

1 Barnard E. A, Miledi R., Sumikawa K., Translation of exogenous messenger RNA coding for nicotinic acetylcholine receptors produces functional receptors in Xenopus oocytes, Proc. R. Soc. London 1982, 215, 241-246.

2 Gundersen C. B., Miledi R., Parker I., Messenger RNA from human brain induces drug- and voltage- operated chan- nels in Xenopus oocytes, Nature 1984, 308, 421-424.

3 Miledi R., Parker I., Sumikawa K., Properties of acetylcholine receptors translated by cat muscle mRNA in Xenopus oocytes, EMBO J. 1982, 1, 1307-1312.

4 Methfessel C., Witzemann V., Takahashi T., Mishina M., Numa S., SaKMANN B., Patch clamp measurements on 
Xenopus laevis oocytes: currents through endogenous channels and implanted acetylcholine receptor and sodium channels, Pflügers Arch. 1986, 407, 577-588.

5 Thornhill W. B., Levinson S. R., Biosynthesis of electroplax sodium channels in Electrophorus electrocytes and Xenopus oocytes, Biochemistry 1987, 26, 43814388.

6 Thornhill W. B., Levinson S. R., Biosynthesis of electroplax sodium channels, Ann. N. Y. Acad. Sci. 1986, 479, 356-363.

7 Lübbert H., Hoffman B. J., SnUtch T. P.,VAnDyke T., Levine A. J., Hartig P. R., Lester H. A., Davidson N., cDNA cloning of a serotonin $5-\mathrm{HT}_{1 \mathrm{C}}$ receptor by electrophysiological assays of mRNAinjected Xenopus oocytes, Proc. Natl. Acad. Sci. USA 1987, 84, 4332-4336.

8 Julius D., MacDermott A. B., Axel R., Jessell T. M., Molecular characterization of a functional cDNA encoding the serotonin $1 \mathrm{c}$ receptor, Science 1988, 241, 558564.

9 WeBer W-M., Ion currents of Xenopus laevis oocytes: state of the art, Biochim. Biophys. Acta 1999, 1421, 213-233.

10 Taglialatela M., Toro L., Stefani E., Novel voltage clamp to record small, fast currents from ion channels expressed in Xenopus oocytes, Biophys. J. 1992, 61, 78-82.

11 Stefani E., Bezanilla F., Cut-open oocyte voltage-clamp technique, Methods Enzymol. 1998, 293, 300-318.

12 Goldin A. L., Maintenance of Xenopus laevis and oocyte injection, Methods Enzymol. 1992, 207, 266-279.

13 Goldin A. L., Sumikawa K., Preparation of RNA for injection into Xenopus oocytes, Methods Enzymol. 1992, 207, 279297.

14 SтÜнmer W., Electrophysiologic recordings from Xenopus oocytes, Methods Enzymol. 1998, 293, 280-300.

15 Schreibmayer W., Lester H.A., Dascal N., Voltage clamping of Xenopus laevis oocytes utilizing agarose-cushion electrodes, Pflügers Arch. 1994, 426, 453-458.

16 Stühmer W., Methressel C., Sakmann B., Noda M., Numa S., Patch clamp characterization of sodium channels expres- sed from rat brain cDNA, Eur. Biophys. J. 1987, 14, 131-138.

17 YANG X-C., SACHs F., Characterization of stretch-activated ion channels in Xenopus oocytes, J. Physiol.(London) 1990, 431, 103-122.

18 Goldin, A. L., Expression of ion channels by injection of mRNA into Xenopus oocytes, Methods Cell Biol. 1991, 36, 487509.

19 Shiн, T. M., Sмith, R. D., Toro, L., GolDIN, A. L., High-level expression and detection of ion channels in Xenopus oocytes, Methods Enzymol. 1998, 293, 529-556.

20 Schmidt J. W., Rossie S., Catterall W. A., A large intracellular pool of inactive Na channel alpha subunits in developing rat brain, Proc. Natl. Acad. Sci. USA 1985, 82, 4847-4851.

21 Colman A., Translation of eukaryotic messenger RNA in Xenopus oocytes, 1984, 1, 271-302.

22 Zhou W., Chung I., Liu Z., Goldin A. L., Dong K., A voltage-gated calciumselective channel encoded by a sodium channel-like gene, Neuron 2004, 42, 101112.

23 Liu Z., Chung I., Dong K, Alternative splicing of the $B S C 1$ gene generates tissue-specific isoforms in the German cockroach, Insect Biochem. Mol. Biol. 2001, 31, 703-713.

24 Salkoff L., Butler A., Wei A., Scavarda N., Giffen K., Ifune C., Goodman, R., MANDEL G. Genomic organization and deduced amino acid sequence of a putative sodium channel gene in Drosophila, Science 1987, 237, 744-748.

25 Stühmer W., Conti F., Suzuki H., Wang X., Noda M., Yahagi N., Kubo H., Numa, S. Structural parts involved in activation and inactivation of the sodium channel, Nature 1989, 339, 597-603.

26 Papazian D. M., Timpe L.C., Jan Y.N., JAN L.Y., Alteration of voltage-dependence of Shaker potassium channel by mutations in the S4 sequence, Nature 1991, 349, 305-310.

27 Hoshi T., Zagotta W. N., Aldrich R.W., Biophysical and molecular mechanisms of Shaker potassium channel inactivation, Science 1990, 250, 533-538. 
28 Zagotta W. N., Hoshi T., Aldrich R.W., Restoration of inactivation in mutants of Shaker potassium channels by a peptide derived from ShB, Science $\mathbf{1 9 9 0}$, 250, 568-571.

29 Patton D. E., West J. W., Catterall W. A., Goldin, A. L., Amino acid residues required for fast sodium channel inactivation. Charge neutralizations and deletions in the III-IV linker. Proc. Natl. Acad. Sci. USA 1992, 89, 10905-10909.

30 West J.W., Patton D. E., Scheuer T., Wang Y., Goldin A. L, Catterall W. A., A cluster of hydrophobic amino acid residues required for fast $\mathrm{Na}^{+}$channel inactivation, Proc. Natl. Acad. Sci. USA 1992, 89, 10910-10914.

31 Yellen G., Jurman M. E., Abramson T., MacKinnon R., Mutations affecting internal TEA blockade identify the probable pore-forming region of a $\mathrm{K}^{+}$channel, Science 1991, 251, 939-942.

32 MacKinnon R., Miller C., Mutant potassium channels with altered binding of charybdotoxin, a pore-blocking inhibitor, Science 1989, 245, 1382-1385.

33 MacKinnon R., Yellen G., Mutations affecting TEA blockade and ion permeation in voltage-activated $\mathrm{K}^{+}$channels, Science 1990, 250, 276-279.

34 MacKinnon R., Heginbotham L., Abramson T., Mapping the receptor site for a pore-blocking potassium channel inhibitor, Neuron 1990, 5, 767-771.

35 Yool A. J., Schwarz T. L., Alteration of ionic selectivity of a $\mathrm{K}^{+}$channel by mutation of the H5 region, Nature 1991, 349, 700-704.

36 Hartmann H. A., Kirsch G. E., Drewe J. A., Taglialatela M., Joho R. H., Brown, A. M., Exchange of conduction pathways between two related $\mathrm{K}^{+}$channels, Science 1991, 251, 942-944.

37 Javitch, J. A., Fu, D., Chen, J., Karlin, A., Mapping the binding-site crevice of the dopamine D2 receptor by the substituted-cysteine accessibility method, Neuron 1995, 14, 825-831.

38 Cha, A., Snyder, G. E., Selvin, P. R., Bezanilla, F., Atomic scale movement of the voltage-sensing region in a potassium channel measured via spectroscopy, Nature 1999, 402, 809-813.
39 Mannuzzu, L. M., Isacoff, E. Y. Independence and cooperativity in rearrangements of a potassium channel voltage sensor revealed by single subunit fluorescence, J. Gen. Physiol. 2000, 115, 257268.

40 Gandhi, C. S., Clark, E., Loots, E., Pralle, A., Isacoff, E. Y., The orientation and molecular movement of a $\mathrm{K}^{+}$channel voltage-sensing domain, Neuron 2003, 40, 515-525.

41 Jiang, Y., Lee, A., Chen, J., Ruta,V., Cadene, M., Chait, B. T., MacKinnon, R., X-ray structure of a voltage-dependent $\mathrm{K}^{+}$channel, Nature 2003, 423, 33-41.

42 Jiang, Y., Ruta, V., Chen, J., Lee, A., MACKInNON, R., The principle of gating charge movement in a voltage-dependent $\mathrm{K}^{+}$channel, Nature 2003, 423, 42-48.

43 Chanda, B., Bezanilla, F., Tracking voltage-dependent conformational changes in skeletal muscle sodium channel during activation, J. Gen. Physiol. 2002, 120, 629-645.

44 Chanda, B., Asamoah, O. K., BeZANilla, F., Coupling interactions between voltage sensors of the sodium channel as revealed by site-specific measurements, J. Gen. Physiol. 2004, 123 , 217-230.

45 Sonnleitner, A., Mannuzzu, L. M., Terakawa, S., Isacoff, E. Y., Structural rearrangements in single ion channels detected optically in living cells, Proc. Natl. Acad. Sci. USA 2002, 99, 1275912764.

46 Asamoah, O. K., Wuskell, J. P., Loew, L. M., Bezanilla, F. A., Fluoremetric approach to local electric field measurements in a voltage-gated ion channel, Neuron 2003, 37, 85-97.

47 Cohen, B. E., Pralle, A., Yao, X. J., Swaminath, G., Gandhi, C. S., Jan, Y. N., Кobilka, B. K., IsAcoff, E. Y. ET AL., A fluorescent probe designed for studying protein conformational change, Proc. Natl. Acad. Sci. USA 2005, 102, 965-970.

48 Cannon, S. C., From mutation to myotonia in sodium channel disorders. Neuromusc. Disord. 1997, 7, 241-249.

49 Cannon, S. C., Spectrum of sodium channel disturbances in the nondystro- 
phic myotonias and periodic paralyses, Kidney Int. 2000, 57, 772-779.

50 BALSER, J. R., Inherited sodium channelopathies: models for acquired arrhythmias? Am. J. Physiol. Heart Circ. Physiol. 2002, 282, H1175-H1180.

51 Escayg, A., MacDonald, B. T., Meisler, M. H., Baulac, S., Huberfeld, G., An-Gourfinkel, I., Brice, A., LeGuern, E. ET AL., Mutations of SCN1A, encoding a neuronal sodium channel, in two families with GEFS+2, Nat. Genet. 2000, 24, 343-345.

52 Escayg, A., Heils, A., MacDonald, B. T., Haug, K., Sander, T., Meisler, M. H., A novel SCN1A mutation associated with generalized epilepsy with febrile seizures plus and prevalence of variants in patients with epilepsy, Am. J. Hum. Genet. 2001, 68, 866-873.

53 Wallace, R. H., Scheffer, I. E., Barnett, S., Richards, M., Dibbens, L., Desai, R. R., Lerman-Sadie, T., Lev, D. et al., Neuronal sodium-channel a1-subunit mutations in generalized epilepsy with febrile seizures plus, Am. J. Hum. Genet. 2001, 68, 859-865.

54 Sugawara, T., Tsurubuchi, Y., Agarwala, K. L., Ito, M., Fukuma, G., Mazaki-Miyazaki, E., Nagafuji, H., NodA, M. ET AL., A missense mutation of the Na+ channel aII subunit gene Nav1.2 in a patient with febrile and afebrile seizures causes channel dysfunction, Proc. Natl. Acad. Sci. USA 2001, 98, 63846389.

55 Wallace, R. H., Wang, D. W., Singh, R., Scheffer, I. E., George, A. L., Jr. Phillips, H. A., SAAr, K., Reis, A. et al., Febrile seizures and generalized epilepsy associated with a mutation in the $\mathrm{Na}^{+}$channel b1 subunit gene SCN1B, Nat. Genet. 1998, 19, 366-370.

56 Wallace, R. H., Scheffer, I. E., Parasivam, G., Barnett, S., Wallace, G. B., Sutherland, G. R., Berkovic, S. F., Mulley, J. C., Generalized epilepsy with febrile seizures plus: mutation of the sodium channel subunit SCN1B, Neurology 2002, 58, 1426-1429.

57 Bendahhou, S., Cummins, T. R., Hahn, A. F., LANGlois, S., WaXman, S. G., Ptácek, L. J., A double mutation in fami- lies with periodic paralysis defines new aspects of sodium channel slow inactivation, J.Clin.Invest. 2000, 106, 431-438.

58 Struyk, A. F., Scoggan, K. A., Bulman, D. E., CAnnon, S. C., The human skeletal muscle Na channel mutation $\mathrm{R} 669 \mathrm{H}$ associated with hypokalemic periodic paralysis enhances slow inactivation, J.Neurosci. 2000, 20, 8610-8617.

59 Featherstone, D. E., Fujimoto, E., Ruben, P. C., A defect in skeletal muscle sodium channel deactivation exacerbates hyperexcitability in human paramyotonia congenita, J. Physiol. (London) 1998, 506, 627-638.

60 Cannon, S. C., Brown, R. H., Jr. Corey, D. P., Theoretical reconstruction of myotonia and paralysis caused by incomplete inactivation of sodium channels, Biophys. J. 1993, 65, 270-288.

61 Chen, Q., Kirsch, G. E., Zhang, D., Brugada, R., Brugada, J., Brugada, P., Potenza, D., Moya, A. ET AL., Genetic basis and molecular mechanism for idiopathic ventricular fibrillation, Nature 1998, 392, 293-296.

62 Rook, M. B., Alshinawi, C. B., GroeneWEGEN, W. A., van Gelder, I. C., vaN Ginneken, A. C. G., Jongsma, H. J., Mannens, M. M. A. M., Wilde, A. A. M., Human SCN5A gene mutations alter cardiac sodium channel kinetics and are associated with the Brugada syndrome, Cardiovasc. Res. 1999, 44, 507-517.

63 Baroudi, G., Carbonneau, E., Pouliot, V., Chahine, M. SCN5A mutation (T1620M) causing Brugada syndrome exhibits different phenotypes when expressed in Xenopus oocytes and mammalian cells, FEBS Lett. 2000, 467, 12-16.

64 Abriel, H., Cabo, C., Wehrens, X. H. T., Rivolta, I., Motoike, H. K., Memmi, M., Napolitano, C., Priori, S. G. et al., Novel arrhythmogenic mechanism revealed by a long-QT syndrome mutation in the cardiac $\mathrm{Na}^{+}$channel, Circ. Res. 2001, 88, 740-745.

65 Papadatos, G. A., Wallerstein, P. M. R., Head, C. E. G., Ratcliff, R., Brady, P. A., Benndorf, K, Saumarez, R. C., Trezise, A. E. O. ET AL., Slowed conduction and ventricular tachycardia after targeted disruption of the cardiac sodium 
channel gene Scn5a, Proc. Natl. Acad. Sci. USA 2002, 99, 6210-6215.

66 Spampanato, J., Escayg, A., Meisler, M. H., Goldin, A. L., Functional effects of two voltage-gated sodium channel mutations that cause generalized epilepsy with febrile seizures plus type 2, J. Neurosci. 2001, 21, 7481-7490.

67 Spampanato, J., Escayg, A., Meisler, M. H., Goldin, A. L., The generalized epilepsy with febrile seizures plus type 2 mutation W1204R alters voltage-dependent gating of $\mathrm{Na}_{\mathrm{v}} 1.1$ sodium channels, Neuroscience 2003, 116, 37-48.

68 Alekov, A. K., Rahman, M. M., Mitrovic, N., Lehmann-Horn, F., LERCHE, H., A sodium channel mutation causing epilepsy in man exhibits defects in fast inactivation and activation in vitro, J. Physiol.(London) 2000, 529, 533-539.

69 Alekov, A. K., Rahman, M., Mitrovic, N., Lehmann-Horn, F., Lerche, H., En- hanced inactivation and acceleration of activation of the sodium channel associated with epilepsy in man, Eur. J. Neurosci. 2001, 13, 2171-2176.

70 Lossin, C., WanG, D. W., Rhodes, T. H., Vanoye, C. G., George, A. L., Jr., Molecular basis of an inherited epilepsy, Neuron 2002, 34, 877-884.

71 Lossin, C., Rhodes, T. H., Desai, R. R., Vanoye, C. G., Wang, D., Carniciu, S., Devinsky, O., George, A. L., JR., Epilepsy-associated dysfunction in the voltage-gated neuronal sodium channel SCN1A, J. Neurosci. 2003, 23, 1128911295.

72 Spampanato, J., Kearney, J. A., de HaAn, G., McEwen, D. P., Escayg, A., Aradi, I., MacDonald, B. T., Levin, S. I. et al., A novel epilepsy mutation in the sodium channel SCN1A identifies a cytoplasmic domain for b subunit interaction, J. Neurosci. 2004, 24, 10022-10034. 